\title{
GC-MS Analysis and Molecular Docking Studies of Active Phytochemicals from Medicinal Plants against Malassezia Globosa LIP1 (SMG1) Enzyme
}

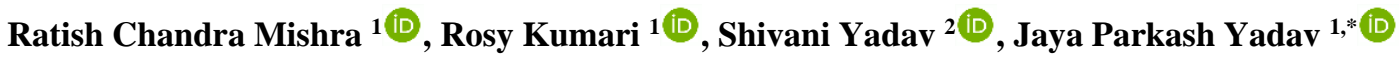 \\ 1 Plant Genetics Lab; Department of Genetics; Maharshi Dayanand University; Rohtak-124001; Haryana; India; \\ rcmishra0001@gmail.com (R.C.M.); rosyaryanature@gmail.com (R.K.); yadav1964@ rediffmail.com (J. P. Y.); \\ 2 Department of Computer Science and Application; Maharshi Dayanand University; Rohtak-124001; Haryana; India; \\ shivaniyadav17@gmail.com (S.Y.); \\ Correspondence: yadav1964@rediffmail.com (J. P. Y.);
}

Scopus Author ID 7201786885

Received: 14.05.2021; Revised: 25.06.2021; Accepted: 1.07.2021; Published: 8.08.2021

\begin{abstract}
Malassezia globosa LIP1 (SMG1) lipase plays a crucial role in the pathogenicity of $M$. globosa in dandruff sufferers. In this study, GC-MS analysis of selected medicinal plants was done, and antifungal activity of these identified phytochemicals was checked by molecular docking method against Malassezia globosa LIP1 (SMG1) lipase using AutoDock 4.0. SwissADME tool was used to analyze the absorption, distribution, metabolism, excretion (ADME) of phytoligands. GC-MS showed various phytoconstituents in the Glycyrrhiza glabra extract, including glabridin, 2-propenal, 3-phenyleugenol, 4'-O-methylglabridin, hispaglabridin A, stigmast-5-en-3-ol, stigmasta-5,3-dien-7-one, glabrol. Punica granatum extract contains hydroxymethylfurfural, stigmast-5-en-3-ol, 4h-pyran-4-one, 2,3dihydro-3,5-dihydroxy-6-methyl-, 1,2,3-benzenetriol, d-glucopyranose, 1,6-anhydro- as the major compounds. Among one hundred thirty screened compounds, twenty-one followed Lipinski's rule of five and were nontoxic in nature. Docking results reveal that among all, $\beta$-sitosterol, stigmatsa-5,3-dien7-one, glabrol and 22,23-dibromostigmast-5-en-3-yl acetate (DBSA) showed the highest binding affinity with SMG1 i.e. $-6.29 \mathrm{kcal} / \mathrm{mol},-6.50 \mathrm{kcal} / \mathrm{mol},-10.12 \mathrm{kcal} / \mathrm{mol}$ and $-11.04 \mathrm{kcal} / \mathrm{mol}$ respectively as compared to standard inhibitor RHC $80267(-5.83 \mathrm{kcal} / \mathrm{mol})$. From the results, we conclude that the lead compounds may be used as potential anti-dandruff agents. Plant-based antidandruff products are eco-friendly and considered a safe alternative due to their less or negligible side effects.
\end{abstract}

Keywords: anti-dandruff; Malassezia globosa; SMG1; lipase;molecular docking; ADME; GC-MS

(C) 2021 by the authors. This article is an open-access article distributed under the terms and conditions of the Creative Commons Attribution (CC BY) license (https://creativecommons.org/licenses/by/4.0/).

\section{Introduction}

Malassezia globosa is considered to be the main causative microbe for dandruff infection [1]. The fungus proliferates through Malassezia globosa LIP1 (SMG1), which is a monoacylglycerol (MAG) and diacylglycerol (DAG) lipase [2,3]. The enzyme produces saturated and unsaturated free fatty acids (FFAs) during lipid metabolism. Unsaturated FFA participates in fungal pathogenicity and induces skin inflammation, flaking, and itching [4-6]. Therefore, SMG1 can be a potential target to inhibit fungal growth. Nowadays, various antifungal agents are used for management of dandruff such as zinc pyrithione, ketoconazole, zinc pyrithione, chlorhexidine, miconazole, piroctone olamine, triclosan, selenium sulfide, miconazole, coal tar, azole compounds (fluconazole, voriconazole, itraconazole, posaconazole, 
climbazole, pramiconazole), RHC80267and lipase inhibitors etc. [7]. Due to their various side effects, nontoxic target-based herbal antifungal drugs are needed to develop [8,9]. Medicinal plants such as Cinnamomum zeylanicum, Glycyrrhiza glabra, and Punica granatum contain various bioactive compounds that can be used for this purpose. Cinnamomum zeylanicum Blume (Cinnamon/Dalchini; family Lauraceae) is a small, tropical evergreen tree of south Asia and native to Sri Lanka. Its bark is extensively used and is amply mentioned in Ayurveda for various remedies [10]. Essential oils obtained from cinnamon bark reported antifungal activity against Malassezia spp [11]. It contains compounds, namely eugenol, cinnamaldehyde, $\beta$ caryophyllene, linalool, cinnamyl acetate, eugenyl acetate, (E)-ethyl cinnamate, camphor, linalool, and piperitone [12]. Glycyrrhiza glabra, commonly named as "Licorice/Mulethi/Yashtimadhu" in India, belongs to the family Fabaceae. Aqueous extract of G. glabra root is a healthy wash for falling and graying hair and is used in shampoo formulations $[13,14]$. The plant root contains different bioactive compounds, such as glycyrrhizin, glycirrhizic acid, glabra, glabridin, galbrene, glabrone, 4'-o-methylglabridin [15]. Licorice showed various pharmacological activities [16]. Punica granatum L. belonging to the family Punicaceae is well-known as pomegranate (Anar). Its fruit peels constitute approximately $60 \%$ of the total weight of the pomegranate fruit. Peel is considered an agrowaste, but it can be a potential antimicrobial source $[17,18]$. The dry content is composed of simple sugars (30-35\%), phenolic compounds (10-20\%), and polysaccharides (10-15\%), while the fresh peel contains a large percentage of water (around 70-75\%). The peel contains compounds including gallotannins, gallic acid, ellagic acid, ellagitannins (such as punicalagins and punicalins), Anthocyanins (such as cyanidin and delphinidin glucosides, and numerous gallagyl esters [19-21].

Nowadays, GC-MS analysis has become a key technique for the identification of secondary metabolites in medicinal plants [22]. Plant metabolites display more "drug-likeness and biological activity than fully synthetic compounds", making them superior candidates as a drug [23]. Structure-based drug design can be used to reduce the ambiguity involved and fasten up the process for drug development [24]. Very few molecular docking studies of SMG1 inhibitors of $M$. globosa have been reported. Therefore in this study, virtual screening of selected phytoligands was done to check their binding affinity for SMG1 lipase compared to standard drug RHC 80267.

\section{Materials and Methods}

\subsection{Plant sample.}

Plant materials such as rhizome of Glycyrrhiza glabra, the bark of Cinnamomum zeylanicum, and fruit peel of Punica granatum were collected from the local market of Rohtak city of Haryana, India. The samples were further identified with the help of the flora of Haryana and comparing the herbarium specimens available in the Department of Genetics, Maharshi Dayanand University, Rohtak, Haryana, India.

\subsection{Preparation of extracts.}

Plant samples were shade dried and powdered. $10 \mathrm{~g}$ of prepared sample were dissolved in $100 \mathrm{ml}$ solvent (methanol and ethyl acetate) and rotated in incubator shaker for $48 \mathrm{hrs}$. It was then filtered, lyophilized, and stored for further use. 


\subsection{GC-MS analysis.}

Gas Chromatography-Mass Spectrometry (GC-MS) with nonpolar column Rxi-5Sil MS (30m $\times 0.25 \mathrm{~mm}$ Id $\times 0.25 \mu \mathrm{m}$ film thickness) was performed at AIRF JNU, New Delhi. The method of electron-impact ionization was applied with the following GC-MS conditions (Table 1) $[25]$.

Table 1. Showing GC-MS analysis conditions.

GC-MS conditions

\begin{tabular}{l|l}
\hline Column Oven Temp. : $50.0{ }^{\circ} \mathrm{C}$ & Purge Flow $: 3.0 \mathrm{~mL} / \mathrm{min}$ \\
\hline Ion Source Temp $: 230.00{ }^{\circ} \mathrm{C}$ & Flow Control Mode $:$ Linear Velocity \\
\hline Injection Temp. $: 260.00{ }^{\circ} \mathrm{C}$ & Linear Velocity $: 40.1 \mathrm{~cm} / \mathrm{sec}$ \\
\hline Split Ratio $: 10.0$ & Total Flow $: 16.4 \mathrm{~mL} / \mathrm{min}$ \\
\hline Pressure $: 69.8 \mathrm{kPa}$ & Column Flow $: 1.22 \mathrm{~mL} / \mathrm{min}$
\end{tabular}

\subsection{ADME Analysis.}

SwissADME web tool was used for ADME evaluation [26]. Canonical smiles of molecules were retrieved from PubChem, entered in SwissADME, and run to evaluate pharmacokinetics and toxicity of phytochemicals.

\subsection{Molecular docking analysis.}

The X-ray crystal structure of the SMG1 (PDB Id-3UUE) was retrieved from the Protein Data Bank (www.rcsb.org). SPDBV-Swiss-PdbViewer tool was used to generate model protein for docking. Ligand structures were drawn in Chemsketch, saved as mdl mol file and then converted into pdb by Open Babel 18.0. AutoDock 4 version 1.56rC3 with the Lamarckian genetic algorithm was used, and the parameter was set to $100 \mathrm{GA}$ runs for each docking simulation up to 250,000 energy evaluations [27-29]. Bond orders were assigned, hydrogen atoms were added, and the water molecules that did not interact were removed. The coordinate axes of active protein whereas follow X: -3.594, Y: 6.002, and Z: 2.234. 10 runs were performed at each site, and the poses were clustered by heavy atom root-mean-square deviation (RMSD), and the value of the docking score was calculated.

\subsection{Visualization.}

The best docking complexes were examined by UCSF Chimera. The tool provided the visualization of the interaction between ligands and amino acids of the targeted protein.

\subsection{Reference strains and chemicals.}

Pathogenic reference viz. Malassezia furfur MTCC 1374 was used for the study. Strains were procured from IMTECH, Chandigarh (India). Glabrol, DBSA, stigmatsa-5,3-dien,7-one, $\beta$-sitosterol, and RHC-80267were purchased from Sigma (St. Louis, USA).

\subsection{Antifungal activity.}

Antifungal activity of the lead compound was studied by the agar well diffusion method against Malassezia furfur [22]. RHC-80267 was used as a positive control. 


\subsection{Minimum inhibitory concentration.}

MIC of study samples was calculated using the micro broth dilution method with slight modifications [22]. $2 \mathrm{mg}$ resazurin dye was prepared in $10 \mathrm{~mL}$ of distilled water. Living microbes reduced the dye, and a color change from purple to pink or colorless was observed. In the absence of living microbes, indicator color remained the same. The lowest concentration at which color change occurred was taken as MIC.

\section{Results and Discussion}

\subsection{GC-MS analysis.}

Results revealed that a total of 78 compounds were identified in the G. glabra ethyl acetate extract as depicted in Figure $1 \&$ Table 2. Major phytoconstituents included glabridin with peak area (16.38\%), 3-phenyl-2-propenal (8.07\%), eugenol (4.64\%), 4'-Omethylglabridin (4.1\%), hispaglabridin a (2.67\%), stigmast-5-en-3-ol (2.49\%), stigmasta-3,5dien-7-one (2.39\%), glabrol (2.07\%), cis-dimethyl morpholine (2.54\%), guanosine $(2.42 \%)$.

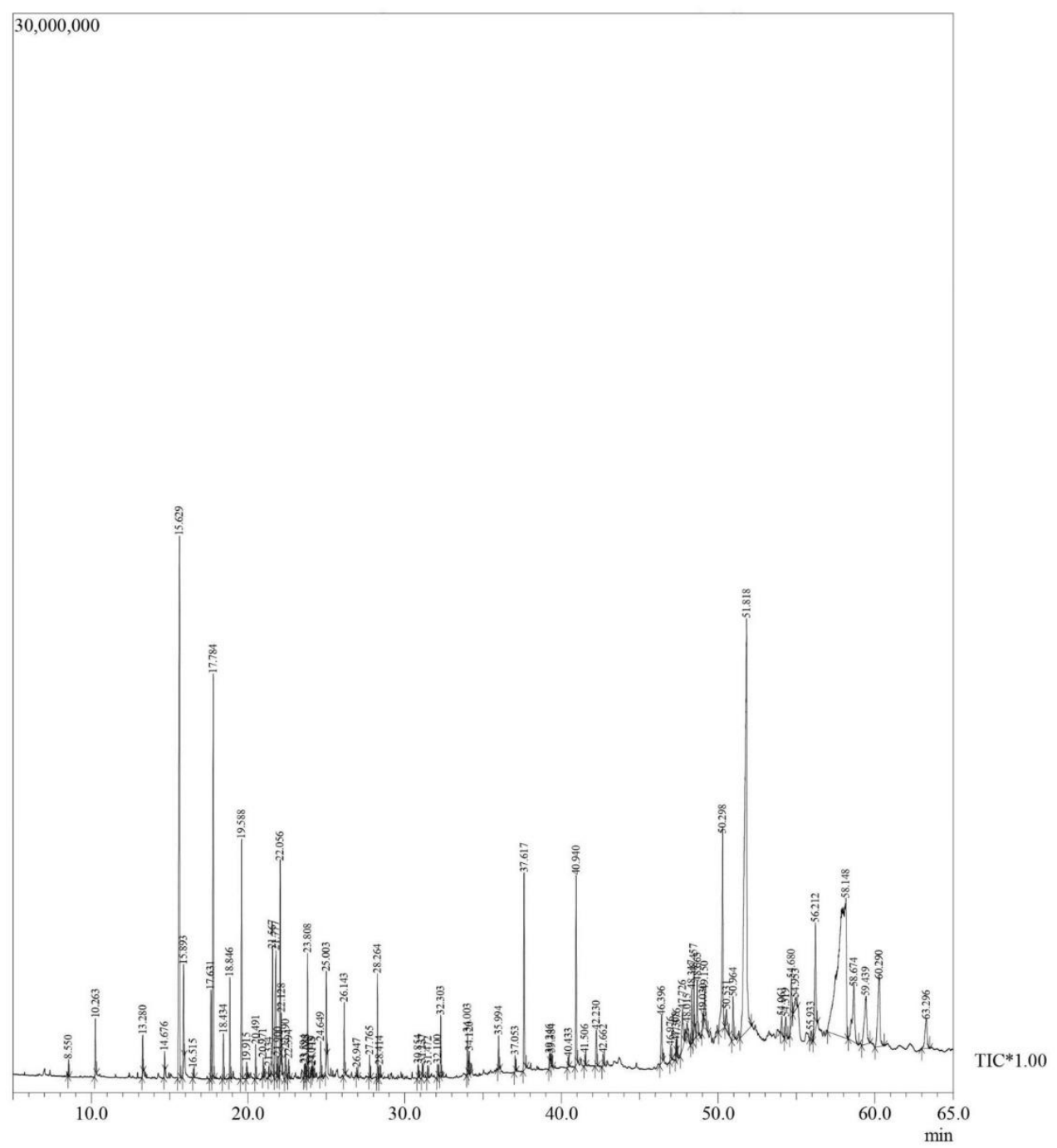

Figure 1. GC-MS spectra of ethyl acetate extract of G. glabra root.

Table 2. GC-MS analysis of G. glabra root extract.

\begin{tabular}{l|l|l|l} 
Peak & R.Time & \% Area & \multicolumn{1}{c}{ Compounds Name } \\
\hline 1 & 8.550 & 0.14 & Eucalyptol (1,8-cineole) \\
\hline 2 & 10.263 & 0.58 & $1,2,3$-Propanetriol, 1-acetate \\
\hline 3 & 13.280 & 0.32 & 1-Dodecene
\end{tabular}




\begin{tabular}{|c|c|c|c|}
\hline Peak & R.Time & $\%$ Area & Compounds Name \\
\hline 4 & 14.676 & 0.20 & 1,2,3-Propanetriol, diacetate \\
\hline 5 & 15.629 & 8.07 & 2-Propenal, 3-phenyl- \\
\hline 6 & 15.893 & 1.25 & Resorcinol \\
\hline 7 & 16.515 & 0.10 & 2-Propen-1-ol, 3-phenyl- \\
\hline 8 & 17.631 & 0.92 & $\alpha$-Terpinyl acetate \\
\hline 9 & 17.784 & 4.64 & Eugenol \\
\hline 10 & 18.434 & 0.42 & Copaene \\
\hline 11 & 18.846 & 0.90 & 1-Tetradecene \\
\hline 12 & 19.588 & 2.32 & $\beta$-Caryophyllene \\
\hline 13 & 19.915 & 0.17 & 2H-1-Benzopyran-2-one \\
\hline 14 & 20.491 & 0.34 & (E,E,E)- 2,6,6,9-tetramethyl-1,4,8-Cycloundecatriene \\
\hline 15 & 20.971 & 0.17 & $\gamma$-Muurolene \\
\hline 16 & 21.334 & 0.09 & Decahydro-4a-methyl-1-methylene-7-(1-methylethenyl)- naphthalene \\
\hline 17 & 21.567 & 1.34 & $\alpha$-Muurolene \\
\hline 18 & 21.777 & 2.22 & 1-Isopropyl-7-methyl-4-methylene-1,2,3,4,4a,5,6,8a-octahydronaphthalene \\
\hline 19 & 21.900 & 0.21 & Phenol, 2-methoxy-4-(2-propenyl)-, acetate \\
\hline 20 & 22.056 & 1.92 & Naphthalene, 1,2,3,5,6,8a-hexahydro-4,7-dimethyl-1-(1-methylethyl)-, (1S-Cis)- \\
\hline 21 & 22.128 & 0.47 & Cis-calamenene \\
\hline 22 & 22.390 & 0.35 & Naphthalene, 1,2,3,4,4a,7-hexahydro-1,6-dimethyl-4-(1-methylethyl)- \\
\hline 23 & 22.594 & 0.21 & $\alpha$-Calacorene \\
\hline 24 & 23.594 & 0.12 & Caryophyllene oxide \\
\hline 25 & 23.688 & 0.13 & Gleenol \\
\hline 26 & 23.808 & 1.14 & 1-Heptadecene \\
\hline 27 & 24.043 & 0.07 & 1-(2,4,5-triethylphenyl)- Ethanone \\
\hline 28 & 24.119 & 0.06 & 1,5,5,8-Tetramethyl-3,7-cycloundecadien-1-ol \\
\hline 29 & 24.649 & 0.37 & Di-epi-1,10-cubenol \\
\hline 30 & 25.003 & 0.91 & 3,4,4-Trimethyl-3-(3-oxo-but-1-enyl)-bicyclo[4.1.0]heptan-2-one \\
\hline 31 & 26.143 & 0.73 & $\gamma$-4-Trimethyl-imidazole-5-butyric acid \\
\hline 32 & 26.947 & 0.08 & (E)-4-(3-Hydroxyprop-1-en-1-yl)-2-methoxyphenol \\
\hline 33 & 27.765 & 0.23 & 6-Tert-butyl-4-methylcoumarin \\
\hline 34 & 28.264 & 0.97 & 1-Heptadecene \\
\hline 35 & 28.414 & 0.15 & Pentadecane \\
\hline 36 & 30.854 & 0.14 & P-Octylacetophenone \\
\hline 37 & 31.137 & 0.13 & Isolongifolen-5-one \\
\hline 38 & 31.472 & 0.14 & 1,2-Benzenedicarboxylic acid, bis(2-methylpropyl) ester \\
\hline 39 & 32.100 & 0.14 & Trans-sinapyl alcohol \\
\hline 40 & 32.303 & 0.60 & 1-Nonadecene \\
\hline 41 & 34.003 & 0.43 & n-Nonadecanol-1 \\
\hline 42 & 34.120 & 0.21 & 9,12-Octadecadienoic acid (Z,Z)-, methyl ester \\
\hline 43 & 35.994 & 0.32 & n-Tetracosanol-1 \\
\hline 44 & 37.053 & 0.17 & 9,12-Octadecadien-1-ol, (Z,Z)- \\
\hline 45 & 37.617 & 2.21 & Behenic alcohol \\
\hline 46 & 39.246 & 0.10 & 5-Hydroxy-2-phenyl-4-chromanone \\
\hline 47 & 39.384 & 0.10 & Behenic alcohol \\
\hline 48 & 40.433 & 0.11 & 9,12-Octadecadien-1-ol, (Z,Z)- \\
\hline 49 & 40.940 & 2.08 & Behenic alcohol \\
\hline 50 & 41.506 & 0.13 & 1,2-Benzenedicarboxylic acid \\
\hline 51 & 42.230 & 0.46 & 6h-Benzofuro[3,2-C][1] benzopyran-3-ol, 6a,11a-dihydro-9 \\
\hline 52 & 42.662 & 0.22 & Oxalic acid, pentadecyl propyl ester \\
\hline 53 & 46.396 & 0.80 & 3,4-Dihydro-3-(2-hydroxy-4-methoxyphenyl)-2h-1-benzopyran-7-ol \\
\hline 54 & 46.976 & 0.21 & Thiazolo[3,2-a]benzimidazol-3(2H)-one, 2-(4-tertbutylbenzylideno)- \\
\hline 55 & 47.308 & 0.13 & Squalene \\
\hline 56 & 47.376 & 0.13 & 2H,6H-Benzofuro[3,2-c]pyrano[2,3-h][1]benzopyran, 6a,11a- \\
\hline 57 & 47.726 & 1.05 & 7-Hydroxy-3-(4-methoxyphenyl)-4H-1-benzopyran-4-one, \\
\hline 58 & 48.015 & 0.28 & 3-Hydroxy-4,4-dimethyl-7-oxoandrost-5-en-17 \\
\hline 59 & 48.317 & 0.59 & Di(pentamethylphenyl)ketone \\
\hline 60 & 48.457 & 0.66 & Decamethylbenzophenone \\
\hline 61 & 48.663 & 0.76 & 17-Methoxy-4-methyl-d-homo-18-norandrosta-4,8,13,15,17 \\
\hline 62 & 49.030 & 0.16 & Bis[3,3,4,7-tetramethyl-1,3-2H-benzofuran-1-yl] ether \\
\hline 63 & 49.150 & 0.59 & 9-(4-Ethoxy-phenyl)-3,4,5,6,7,9-hexahydro-2h-xanthene-1,8-64 \\
\hline 64 & 50.298 & 4.11 & 4'-O-methylglabridin \\
\hline 65 & 50.531 & 0.52 & 1H-Xanthen-1-one, 2,3,4,9-tetrahydro-9-(2-hydroxy \\
\hline 66 & 50.964 & 0.66 & $\beta$-Stigmasta-5,22-dien-3-ol, acetate \\
\hline 67 & 51.818 & 16.38 & Glabridin \\
\hline 68 & 54.061 & 0.32 & $\beta$-Ergost-5-en-3-ol \\
\hline 69 & 54.319 & 0.42 & $\operatorname{Bis}[3,3,4,7$-tetramethyl-1,3-2H-benzofuran-1-yl] ether \\
\hline
\end{tabular}




\begin{tabular}{l|l|l|l} 
Peak & R.Time & \multicolumn{1}{c}{$\%$ Area } & \multicolumn{1}{c}{ Compounds Name } \\
\hline 70 & 54.680 & 0.90 & Stigmasterol \\
\hline 71 & 54.953 & 0.47 & Hispaglabridin B \\
\hline 72 & 55.933 & 0.18 & Bis[3,3,4,7-tetramethyl-1,3-2H-benzofuran-1-yl] ether \\
\hline 73 & 56.212 & 2.49 & $\beta$-Stigmast-5-en-3-ol \\
\hline 74 & 58.148 & 19.96 & No name was assigned to the structure \\
\hline 75 & 58.674 & 2.39 & Stigmasta-3,5-dien-7-one \\
\hline 76 & 59.439 & 2.07 & Glabrol \\
\hline 77 & 60.290 & 2.67 & Hispaglabridin A \\
\hline 78 & 63.296 & 1.11 & N-(3,5-Dichloro-2-hydroxy-4-methylphenyl)-2- butanamide,
\end{tabular}

In the methanol extract of $P$. granatum, 37 bioactives molecules were identified with retention time and $\%$ area as shown in Figure $2 \&$ Table 3 . The main compounds were hydroxymethylfurfural $(60.17 \%)$, stigmast-5-en-3-ol (6.65\%), 4h-pyran-4-one, 2,3-dihydro3,5-dihydroxy-6-methyl- (5.11\%), 1,2,3-benzenetriol (3.53\%), d-glucopyranose, 1,6-anhydro$(3.51 \%)$.

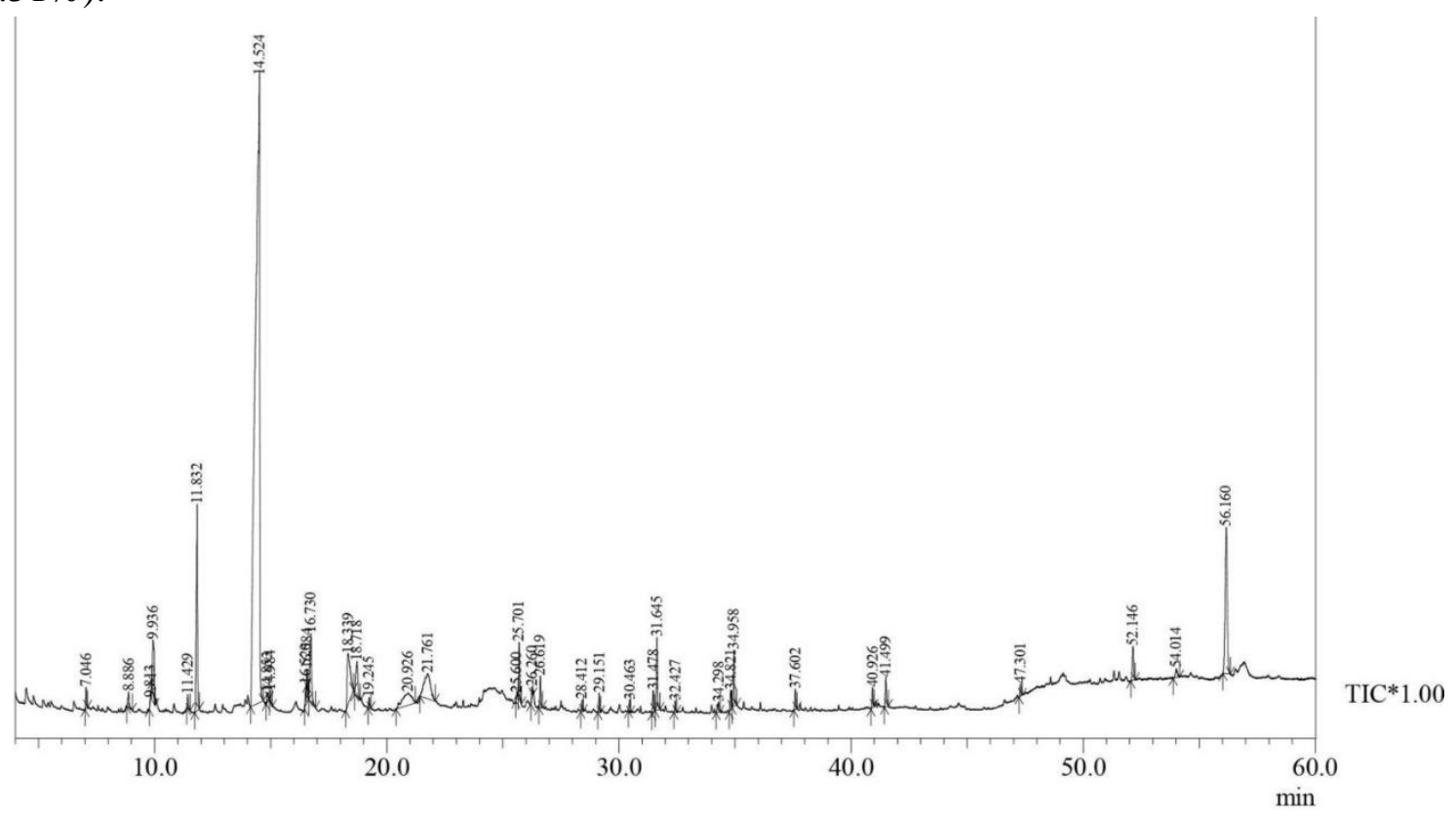

Figure 2. GC-MS spectra of methanol extract of $P$. granatum root.

Table 3. GCMS analysis of $P$. granatum peel extract.

\begin{tabular}{l|l|l|l} 
Peak & R. Time & \%Area & Compounds Name \\
\hline 1 & 7.046 & 0.41 & 2,4-Dihydroxy-2,5-dimethyl-3(2H)-furan-3-one \\
\hline 2 & 8.886 & 0.40 & 2-Nonene, (E)- \\
\hline 3 & 9.813 & 0.04 & 2,5-Furandicarboxaldehyde \\
\hline 4 & 9.936 & 1.75 & Exo-norbornane-2-thiolacetate \\
\hline 5 & 11.429 & 0.30 & 2-Ethyl-5,5-dimethyl-1,3,2-dioxaborolan-4-one \\
\hline 6 & 11.832 & 5.11 & 4H-Pyran-4-one, 2,3-dihydro-3,5-dihydroxy-6-methyl- \\
\hline 7 & 14.524 & 60.17 & 5-Hydroxymethylfurfural \\
\hline 8 & 14.853 & 0.24 & 1,2,3-Propanetriol, 1-acetate \\
\hline 10 & 14.984 & 0.19 & 2,6-Octadien-1-ol, 3,7-dimethyl-, (E)- \\
\hline 11 & 16.520 & 0.20 & Hydrazine, 1,1-diethyl-2-(1-methylpropyl)- \\
\hline 12 & 16.584 & 0.30 & 3-Hydroxy-2-methyl-4H-Pyran-4-one, \\
\hline 13 & 16.730 & 2.54 & CIS-Dimethyl morpholine \\
\hline 14 & 18.339 & 3.53 & 1,2,3-Benzenetriol \\
\hline 15 & 18.718 & 1.51 & Succinic acid, 3-hex-4-ynyl 3-methylbutyl ester \\
\hline 16 & 19.245 & 0.21 & 1,5-Pentandiol, diacetat \\
\hline 18 & 20.926 & 2.42 & Guanosine \\
\hline 19 & 21.761 & 3.51 & $\beta$-D-Glucopyranose, 1,6-anhydro- \\
\hline 20 & 25.600 & 0.11 & Dodecyl isobutyl ether \\
\hline 21 & 25.701 & 1.03 & $\alpha-$ Santalol \\
\hline 22 & 26.260 & 0.22 & Heptadecane \\
\hline
\end{tabular}




\begin{tabular}{l|l|l|l}
\hline Peak & R. Time & \%Area & Compounds Name \\
\hline 24 & 30.463 & 0.23 & Heneicosane \\
\hline 25 & 31.478 & 0.46 & Dibutyl phthalate \\
\hline 26 & 31.645 & 1.76 & n-Hexadecanoic acid \\
\hline 27 & 32.427 & 0.22 & Heneicosane \\
\hline 28 & 34.298 & 0.32 & Heneicosane \\
\hline 29 & 34.821 & 0.42 & $9,12-$ Octadecadienoic acid (Z,Z)- \\
\hline 30 & 34.958 & 1.43 & Oleic Acid \\
\hline 31 & 37.602 & 0.42 & n-Nonadecanol-1 \\
\hline 32 & 40.926 & 0.42 & n-Tetracosanol-1 \\
\hline 33 & 41.499 & 0.69 & 1,2-Benzenedicarboxylic acid \\
\hline 34 & 47.301 & 0.26 & Squalene \\
\hline 36 & 52.146 & 1.00 & Vitamin E \\
\hline 37 & 54.014 & 0.34 & $\beta$-Ergost-5-en-3-ol \\
\hline
\end{tabular}

In our previous article, in C. zeylanicum extract, a variety of phytochemicals were reported such as cinnamaldehyde (75.58\%) and various sesquiterpenes viz. $\beta$-caryophyllene, $\alpha$-copaene, $\alpha$-muurolene, $\alpha$-humulene, $\beta$-bisabolene, cubenol, calamenene, $\alpha$-cadinene, muurolol, cadine-1,4-diene [30].

The principal compound cinnamaldehyde showed antifungal activity against Malassezia pachydermatis [31]. Earlier studies suggested that eugenol has a role in treating skin infections and inhibiting biofilm formation in Malassezia spp [32,33]. $\beta$-Caryophyllene possesses antimicrobial, antifungal, antioxidant, anesthetic, and anti-inflammatory as well as exhibiting anticancerous activity [34,35]. Squalene protects human skin from lipid peroxidation caused by ultraviolet rays [36]. Metabolites, namely 4'-o-methylglabridin, glabridin, squalene, $\beta$-sitosterol, and glabrol inhibited S. epidermidis growth [27]. Quercetin has many health-promoting effects, including anti-inflammatory and anti-allergic effects $[37,38]$. The flavonoid quercetin and kaempferol reported anti-dandruff activity by inhibiting $\beta$-carbonic anhydrase of Malassezia globosa [39]. Essential oil components, namely $\alpha$ copaene, calamenene, $\alpha$-muurolene, $\beta$-bisabolene, muurolol, cubenol, humulene have shown antifungal activity [40-43]. Syringenin showed antioxidant activity [44].

\subsection{ADME analysis.}

Among all 21 compounds were nontoxic in nature, and they followed Lipinski's rule of five (Table 4).

Table 4. ADME analysis of phytoligands.

\begin{tabular}{l|l|l|l|l|l} 
Compounds & $\mathbf{M W}$ & $\begin{array}{l}\text { H-bond } \\
\text { acceptors }\end{array}$ & $\begin{array}{l}\text { H-bond } \\
\text { donors }\end{array}$ & Log $\boldsymbol{P}_{\mathbf{o} / \mathbf{w}}$ & Violation \\
\hline Cinnamaldehyde & 132.16 & 1 & 0 & 1.65 & 0 \\
\hline Eugenol & 164.2 & 2 & 1 & 2.37 & 0 \\
\hline Trans, $\beta$-caryophyllene & 204.35 & 0 & 0 & 3.29 & 1 \\
\hline Squalene & 410.72 & 0 & 0 & 6.37 & 1 \\
\hline 4 '-O- Methylglabridin & 338.4 & 4 & 1 & 3.42 & 0 \\
\hline Glabridin & 324.37 & 4 & 2 & 2.97 & 0 \\
\hline$\beta$-Sitosterol & 414.71 & 1 & 1 & 4.79 & 1 \\
\hline DBSA & 456.74 & 2 & 0 & 5.19 & 1 \\
\hline Stigmatsa-5,3-dien,7-one & 410.67 & 1 & 0 & 4.71 & 1 \\
\hline Glabrol & 392.49 & 4 & 2 & 3.87 & 0 \\
\hline Quecertin & 302.24 & 7 & 5 & 1.63 & 0 \\
\hline Kaempferol & 286.24 & 6 & 4 & 1.7 & 0 \\
\hline$\alpha-$ Copaene & 204.35 & 0 & 0 & 3.4 & 1 \\
\hline$\beta$-Buurolene & 204.35 & 0 & 0 & 3.38 & 1 \\
\hline Cubenol & 204.35 & 0 & 0 & 3.67 & 1 \\
\hline Cyclohex3en1ylmethanol & 222.37 & 1 & 1 & 3.24 & 0
\end{tabular}




\begin{tabular}{l|l|l|l|l|l} 
Compounds & MW & $\begin{array}{l}\text { H-bond } \\
\text { acceptors }\end{array}$ & $\begin{array}{l}\text { H-bond } \\
\text { donors }\end{array}$ & Log $\boldsymbol{P}_{\mathbf{o} / \mathbf{w}}$ & Violation \\
\hline Humulene & 204.35 & 0 & 0 & 3.27 & 1 \\
\hline Calamenene & 202.34 & 0 & 0 & 3.19 & 1 \\
\hline T-Muurolol & 222.37 & 1 & 1 & 3.15 & 0 \\
\hline Syringenin & 372.37 & 9 & 5 & 0.88 & 0
\end{tabular}

The molecule should not have more than $10 \mathrm{H}$-bond acceptors, not have more than 5 H-bond donors, a molecular weight (MW) less than 500 daltons, and an octanol-water partition coefficient, i.e., $\log \mathrm{P}$ not more than $5[45,46]$. Therefore, these molecules were selected to screen out active anti-dandruff agents against lipase enzyme using in silico method.

\subsection{Molecular docking analysis.}

In the present study, active residues Ser171-Asp228-His281 is the catalytic triad of SMG1 lipase was chosen as target [47]. Structures of lead compounds and standard drug RHC80267 are depicted in Figure 3.

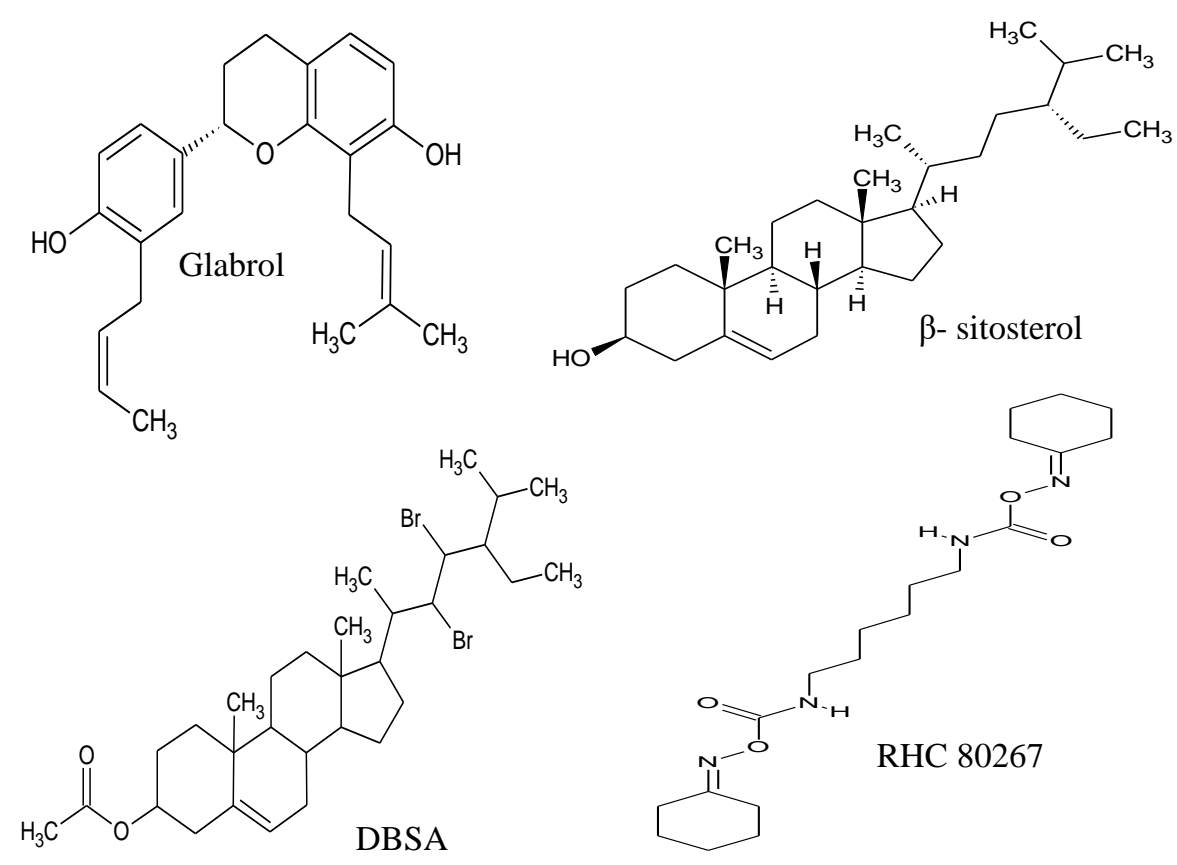

Figure 3. Structure of lead compound and RHC 80267.

Among all, 22,23-dibromostigmast-5-en-3-yl acetate (DBSA) showed the highest binding affinity followed by glabrol, stigmastsa-5,3-dien,7-one, $\beta$-sitosterol with minimum binding energy (MBE) $-11.04,-10.12,6.50,-6.29 \mathrm{kcal} / \mathrm{mol}$, respectively as shown in Table 5 . Reference drug RHC-80267 showed a lower binding affinity with MBE $-5.83 \mathrm{kcal} / \mathrm{mol}$ as compared to lead compounds. Interaction between the enzyme and phytoligands are shown in Table 6 and Figure $4 \& 5$. The protein model (LigPlot+) depicts atomic interactions, such as hydrogen bonds and amino acid interactions, between ligands and atoms in the active site pocket, as shown in Figure 6.

Table 5. Minimum binding energy of selected phytochemicals against SMG1 lipase.

\begin{tabular}{l|l|l} 
S. No. & Compounds Name & MBE against SMG1 (kcal/mol) \\
\hline 1 & Cinnamaldehyde & -4.96 \\
\hline 2 & Eugenol & -4.98 \\
\hline 3 & Trans, $\beta$-caryophyllene & -4.37
\end{tabular}




\begin{tabular}{l|l|l} 
S. No. & Compounds Name & MBE against SMG1 (kcal/mol) \\
\hline 4 & Squalene & -5.19 \\
\hline 5 & 4 '-O- Methylglabridin & -4.88 \\
\hline 6 & Glabridin & -5.13 \\
\hline 7 & $\beta$-Sitosterol & -6.29 \\
\hline 8 & DBSA & -11.04 \\
\hline 9 & Stigmatsa -5,3-dien,7-one & -6.50 \\
\hline 10 & Glabrol & -10.12 \\
\hline 11 & Quecertin & -5.25 \\
\hline 12 & Kaempferol & -5.82 \\
\hline 13 & $\alpha$-Copaene & -4.42 \\
\hline 14 & $\alpha-M u u r o l e n e$ & -4.65 \\
\hline 15 & $\beta$-Bisabolene & -2.00 \\
\hline 16 & Cubenol & -4.65 \\
\hline 17 & Cyclohex3en1ylmethanol & -4.67 \\
\hline 18 & Humulene & -4.45 \\
\hline 19 & Calamenene & -5.75 \\
\hline 20 & T-Muurolol & -4.85 \\
\hline 21 & Syringenin & -3.52 \\
\hline 22 & RHC 80267 & -5.83
\end{tabular}

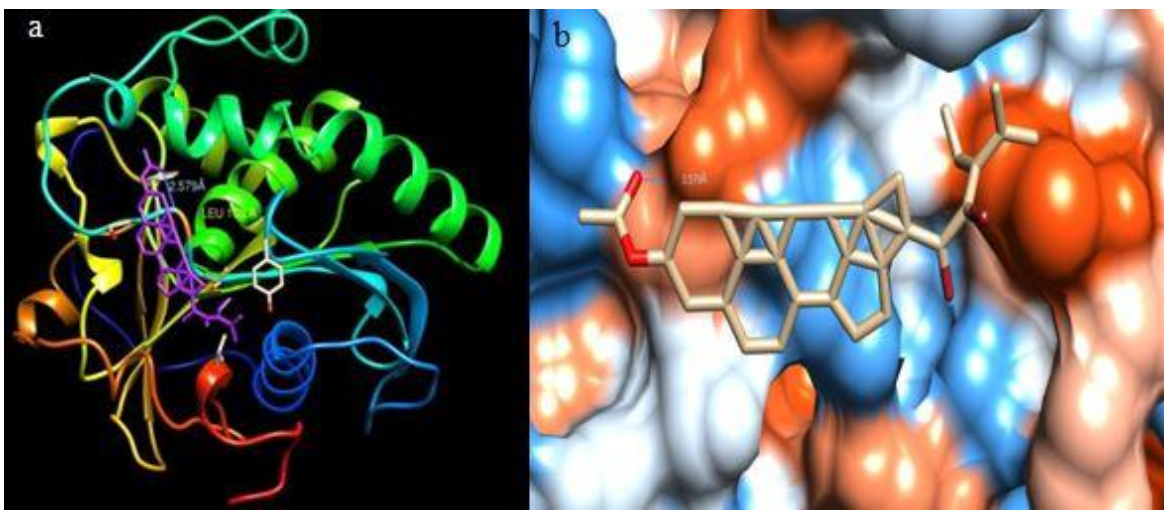

Figure 4. Binding mode of DBSA against SMG1 (a) solid line structure; (b) surface binding mode.

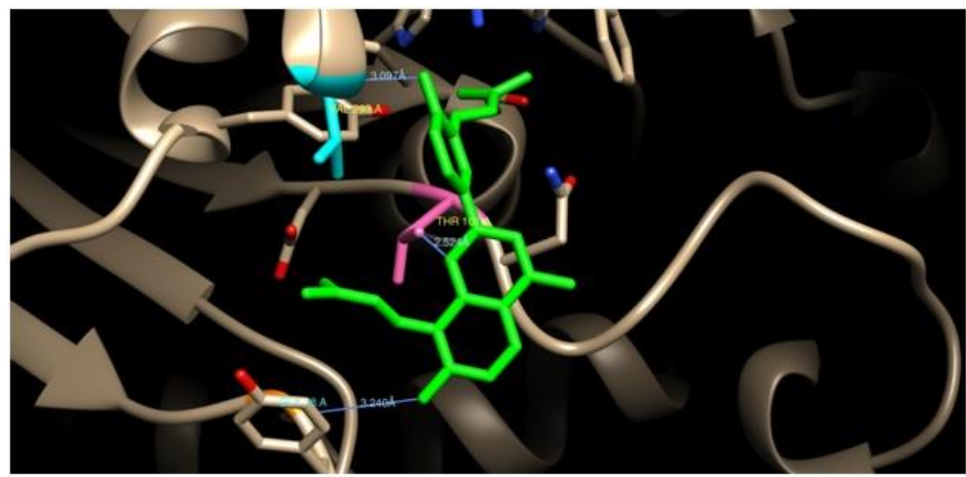

Figure 5. Binding mode of glabrol against SMG1.

Table 6. Ligands protein interaction for lead compounds.

\begin{tabular}{|c|c|c|c|c|}
\hline $\begin{array}{l}\text { Compounds } \\
\text { name }\end{array}$ & MBE & $\begin{array}{l}\text { No. of } \\
\text { H-bond }\end{array}$ & $\begin{array}{l}\text { Amino } \\
\text { involved in } \mathrm{H}- \\
\text { bond }\end{array}$ & Amino acid involve in hydrophobic interaction \\
\hline Glabrol & -10.12 & 3 & $\begin{array}{l}\text { Thr101, Gln282, } \\
\text { Val 293 }\end{array}$ & $\begin{array}{l}\text { Tyr56, His170, Phe278, Ser171, Gly100, His281, } \\
\text { Asn102, Ala292, Glu99, Tyr79, Gly78 }\end{array}$ \\
\hline DBSA & -11.04 & - & - & $\begin{array}{l}\text { Gly78, Thr101, Asn102, Leu103, phe104, Ser105, } \\
\text { Lys114, Val293 }\end{array}$ \\
\hline $\begin{array}{l}\text { Stigmastsa-5,3- } \\
\text { dien,7-one }\end{array}$ & -6.50 & - & - & $\begin{array}{l}\text { Glu77, Gly78, Tyr79, Glu99, Thr101, Asn102, } \\
\text { Leu103, Ser105 and Val293 }\end{array}$ \\
\hline$\beta$-Sitosterol & -6.29 & - & - & $\begin{array}{l}\text { Glu77, Gly78, Tyr79, Glu99, Thr101, Asn102, } \\
\text { Leu103, Ser105 and Val293 }\end{array}$ \\
\hline RHC 80267 & -5.83 & 2 & Thr101, Leu103 & $\begin{array}{l}\text { Glu77, Gly78, Tyr79, Ala80, Glu99,, Asn102, } \\
\text { Phe104, Lys114, Gly137, Ala141 and Val293 }\end{array}$ \\
\hline
\end{tabular}




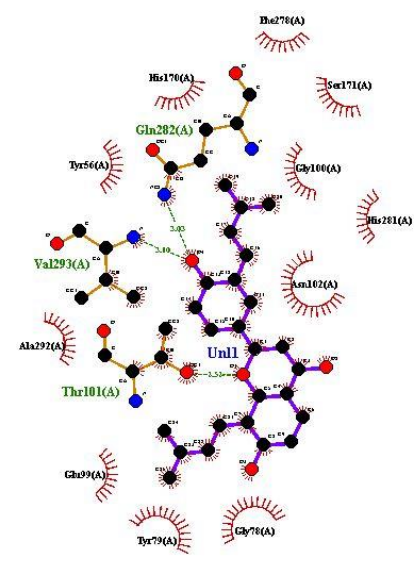

(a) Glabrol

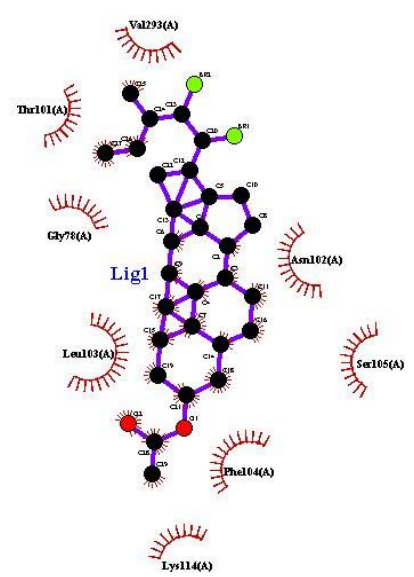

(b) DBSA

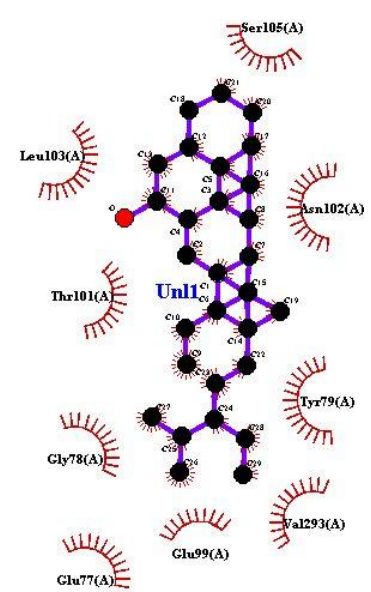

(c) Stigmatsa-5,3-dien, 7-one

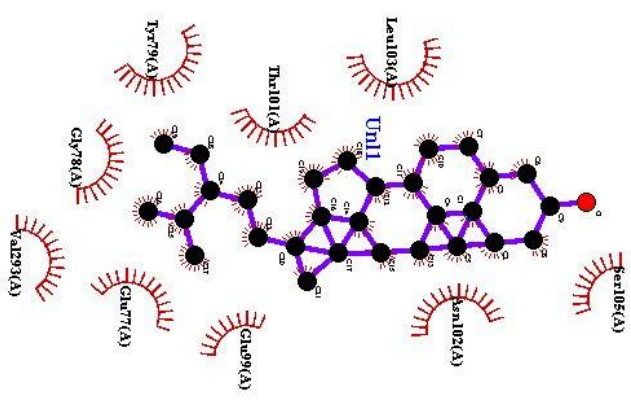

(d) $\beta$-sitosterol

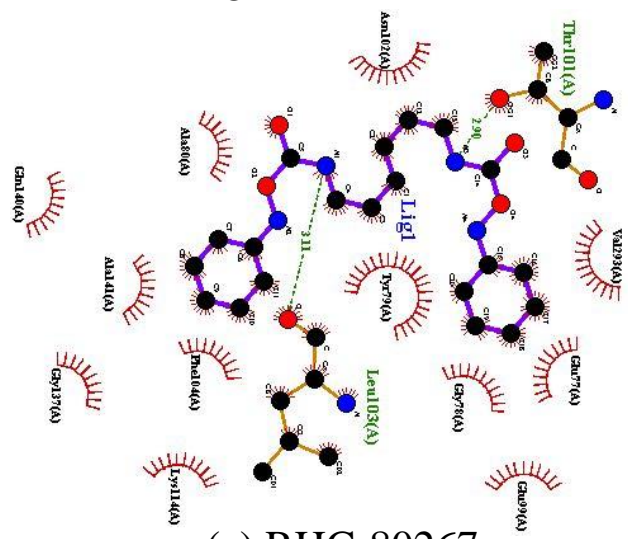

(e) RHC-80267

Figure 6. LigPlot+ to show molecular interaction between lead compounds (a-d) with the amino acid residue of SMG1 protein (3uue). Green dashed lines show H-bonds between ligand and amino acid residue. The spoked arcs represent residues making non-bonded contacts with the ligand. RHC-80267 (e) is a reference drug in this study.

Ligand plot (Figure 6) study clearly shows that active site of 3UUE protein formed 3 hydrogens $(\mathrm{H})$ bonds with glabrol, involving Thr101, Gln282, Val293 with bond length $2.52 \AA$,

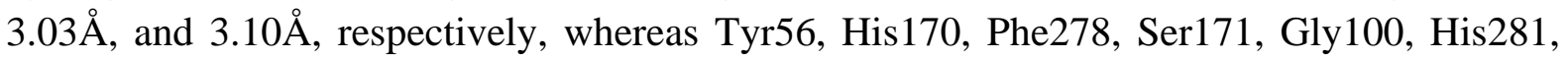
Asn102, Ala292, Glu99, Tyr79, Gly78 participate in hydrophobic interaction; in case DBSA no any H-bond formed and Gly78, Thr101, Asn102, Leu103, phe104, Ser105, Lys114, Val293 are involved hydrophobic interaction with protein 3UUE. Stigmastsa-5,3-dien,7- one formed no H-bond with protein while Glu77, Gly78, Tyr79, Glu99, Thr101, Asn102, Leu103, Ser105, and Val293 formed hydrophobic interaction. $\beta$-Sitosterol formed hydrophobic interaction with Glu77, Gly78, Tyr79, Glu99, Thr101, Asn102, Leu103, Ser105, and Val293 with protein. RHC-80267 standard formed $2 \mathrm{H}$-bond with Thr101, Leu103, and hydrophobic interaction with Glu77, Gly78, Tyr79, Ala80, Glu99,, Asn102, Phe104, Lys114, Gly137, Ala141, and Val293, respectively.

2-(1-Oxo-1,3-dihydro-2H-isoindol-2-yl)ethyl 1-azepanecarbodithioate reported potential inhibitory activity against $M$. globosa LIP1 (SMG1) [47]. Sapondoside-A, kampferol, SNTI inhibit the phospholipase A2 isoform of humans from overcoming dandruff [48]. Compounds such as ZINC85530919, ZINC95914464 reported inhibitory activity for $M$. restricta lipase (Mrlip1) [49]. $\beta$-sitosterol and calceolarioside A inhibits Mala s1 of Malassezia [50]. $\beta$-Sitosterol does not inhibit cytochromes P450 (CYP) enzyme and is also unable to cross the blood-brain barrier (BBB). DBSA exhibited low gastrointestinal absorption, low membrane permeability but did not inhibit CYP. Stigmastsa-5,3-dien,7-one and $\beta$-sitosterol showed 
moderate activity. Glabrol demonstrated high gastrointestinal absorption, high membrane permeability, although it showed inhibitory activity against CYP2C19 and CYP3A4.

\subsection{Antimicrobial activity.}

The antifungal potential of the lead compound such as glabrol, DBSA, stigmastsa-5,3dien,7-one, $\beta$-sitosterol, and standard RHC-80267 was determined against Malassezia furfur using the agar well diffusion method. The zone of inhibition in the range of $12-20 \mathrm{~mm}$. The glabrol showed a maximum zone of inhibition, i.e., $20 \mathrm{~mm}$, followed by DBSA, $\beta$-sitosterol, stigmastsa-5,3-dien,7- one, and standard RHC-80267, i.e., $18 \mathrm{~mm}, 16 \mathrm{~mm}, 16 \mathrm{~mm}$, and $15 \mathrm{~mm}$ respectively.

Minimum inhibitory concentration (MIC) was done by microbroth dilution method. glabrol $(70 \mu \mathrm{g} / \mathrm{mL})$ showed lower MIC as compared to DBSA $(150 \mu \mathrm{g} / \mathrm{mL})$, stigmastsa-5,3dien,7- one $(160 \mu \mathrm{g} / \mathrm{mL}), \beta$-sitosterol $(160 \mu \mathrm{g} / \mathrm{mL})$, and standard RHC-80267 $(100 \mu \mathrm{g} / \mathrm{mL})$ aginst Malassezia furfur.

\section{Conclusions}

The above results provide an insight of phytoligands from $C$. zeylanicum, $P$. granatum, and G. glabra to have an inhibitory effect on Malassezia lipase. SwisADME tool showed that these compounds possess all necessary properties of an ideal drug and follow Lipinski's rule. Therefore, the bioactive compounds including DBSA, glabrol, stigmastsa-5,3-dien,7-one, and $\beta$-sitosterol may be developed as potential anti-dandruff agents.

\section{Funding}

The research was financially supported by UGC under the UGC-SAP program (F.3-20/2012, SAP-II) and UGC BSR fellowships to Ratish Chandra Mishra and Rosy Kumari (F.25-1/201314).

\section{Acknowledgments}

This research has no acknowledgment.

\section{Conflicts of Interest}

The authors declare no conflict of interest.

\section{References}

1. Grimshaw, S.G.; Smith, A.M.; Arnold, D.S.; Xu, E.; Hoptroff, M.; Murphy, B. The diversity and abundance of fungi and bacteria on the healthy and dandruff affected human scalp. Plos One 2019, 14, e0225796,https://doi.org/10.1371/journal.pone.0225796.

2. DeAngelis, Y.M.; Saunders, C.W.; Johnstone, K.R.; Reeder, N.L.; Coleman, C.G.; Kaczvinsky Jr, J.R.; Gale, C.; Walter, R.; Mekel, M.; Lacey, M.P.; Keough, T.W. Isolation and expression of a Malassezia globosa lipase gene, LIP1. J. Invest. Dermatol. 2007, 127, 2138-2146, https://doi.org/10.1038/sj.jid.5700844.

3. Bhat, Y.J.; Latief, I.; Hassan, I. Update on etiopathogenesis and treatment of Acne. Indian J. Dermatol. Venereol. Leprol. 2017, 83, 298-306,https://doi.org/10.4103/0378-6323.199581.

4. Schwartz, J.R.; DeAngelis, Y.M.; Dawson Jr, T.L. Dandruff and seborrheic dermatitis: A head scratcher. Prac. Mod. Hair Sci. 2012, 1, 389-413, https://doi.org/10.13188/2373-1044.1000019.

5. Ramesh, V.; Kumar, K.V.A. Herbally medicated liposomal gel for acne vulgaris. World J. Pharm. Res.2017, 6, 507-529,https://doi.org/10.20959/wjpr201714-9917. 
6. Park, M.; Cho, Y.J.; Lee, Y.W.; Jung, W.H. Understanding the mechanism of action of the anti-dandruff agent zinc pyrithione against Malassezia restricta. Sci. Rep. 2018, 8, 12086, https://doi.org/10.1038/s41598018-30588-2.

7. Angiolella, L.; Carradori, S.; Maccallini, C.; Giusiano, G.T.; Supuran, C. Targeting Malassezia species for novel synthetic and natural antidandruff agents. Curr. Med. Chem. 2017, 24, 2392-2412, https://doi.org/10.2174/0929867324666170404110631.

8. Pingili, M.; Vanga, S.; Raparla, R.K. Antifungal activity of plant extracts against dandruff causing organism Malassezia furfur. Int. J. Bioassays. 2016, 5, 5047-5049, https://doi.org/10.21746/ijbio.2016.11.0010.

9. Mishra, R.C.; Kumari, R.; Yadav, J.P. Comparative study of anti-dandruff efficacy of Punica granatum peel and its biosynthesized silver nanoparticles. J. Bionanosci. 2018, 12, 508-514, https://doi.org/10.1166/jbns.2018.1562.

10. Husain, I.; Ahmad, R..; Chandra, A.; Raza, S.T.; Shukla, Y.; Mahdi, F. Phytochemical characterization and biological activity evaluation of ethanolic extract of Cinnamomum zeylanicum. J. Ethnopharmacol. 2018, 219, 110-116, https://doi.org/10.1016/j.jep.2018.02.001.

11. Donato, R.; Sacco, C.; Pini, G.; Bilia, A.R. Antifungal activity of different essential oils against Malassezia pathogenic species. J. Ethnopharmacol. 2020, 249, 112376, https://doi.org/10.1016/j.jep.2019.112376.

12. Joshi, R.K. Chemical disparity in the oil from leaves of Cinnamomum zeylanicum Blume. Flavour Frag J. 2019, 34, 443-449, https://doi.org/10.1002/ffj.3524.

13. Mishra, R.C.; Kumari, R.; Iqbal, Z.; Rizvi, M.M.A.; Yadav, J.P. Synthesis, characterization, comparative anti-dandruff efficacy and cytotoxicity studies of biosynthesized silver nanoparticles by using Glycyrrhiza glabra root. Adv. Sci. Eng. Med. 2020, 12, 156-162, https://doi.org/10.1166/asem.2020.2482.

14. Azadbakht, M.; Monadi, T.; Esmaeili, Z.; Chabra, A.; Tavakoli, N. Formulation and evaluation of licorice shampoo in comparison with commercial shampoo. J. Pharm. Bioallied Sci. 2018, 10, 208-215, https://doi.org/10.4103/JPBS.JPBS_243_17.

15. Batiha, G.E.; Beshbishy, A.M.; El-Mleeh, A.; Abdel-Daim, M.M.; Devkota, H.P. Traditional uses, bioactive chemical constituents, and pharmacological and toxicological activities of Glycyrrhiza glabra L.(Fabaceae). Biomolecules 2020, 10, 1-19, https://doi.org/10.3390/biom10030352.

16. Fatima, G.; Jabeen, A.; Siddiqui, S.A.; Khalid, M. A review on Glycyrrhiza glabra L. (Aṣl al-Sūs) with unani perspective and modern pharmacology. J. Drug Deliv. Ther. 2019, 9, 736-741, https://doi.org/10.22270/jddt.v9i4-s.3310.

17. Zehra, T.; Ahmed, S.; Zehra, S. Review of characteristic components, traditional and pharmacological properties of Punica granatum. RADS J. Pharm. Pharm. Sci. 2019, 7, 39-52, http://jpps.juw.edu.pk/index.php/jpps/article/view/285/200.

18. Asgary S.; Karimi R.; Joshi T.; Kilpatrick K.L.; Moradi S.; Samimi Z.; Mohammadi E.; Farzaei M.H.; Bishayee A. Effect of pomegranate juice on vascular adhesion factors: A systematic review and metaanalysis. Phytomedicine 2020, 80, 153359, https://doi.org/10.1016/j.phymed.2020.153359.

19. Singh, B.; Singh, J.P.; Kaur, A.; Singh, N. Antimicrobial potential of pomegranate peel: A review. Int. J. Food Sci. Tech. 2018, 54, 959-965, https://doi.org/10.1111/ijfs.13964.

20. Karthikeyan, G.; Vidya, A. Phytochemical analysis, antioxidant and antibacterial activity of pomegranate peel. Res. J.Life Sci. Bioinform. Pharm. Chem. Sci. 2019, 5, 218-231, https://doi.org/10.26479/2019.0501.22.

21. Balli, D.; Cecchi, L.; Khatib, M.; Bellumori, M.; Cairone, F.; Carradori, S.; Zengin, G.; Cesa, S.; Innocenti, M.; Mulinacci, N. Characterization of arils juice and peel decoction of fifteen varieties of Punica granatum L.: A focus on anthocyanins, ellagitannins and polysacch arides. Antioxidants 2020, 9, 1-20, https://doi.org/10.3390/antiox9030238.

22. Kumari, R.; Mishra, R.C.; Yadav, A.; Yadav, J.P. Screening of traditionally used medicinal plants for their antimicrobial efficacy against oral pathogens and GC-MS analysis of Acacia nilotica extract. Indian J. Tradit. Know. 2019, 18, 162-168, http://nopr.niscair.res.in/handle/123456789/45667.

23. George, T.K.; Tomy, A.; Jisha, M.S. Molecular docking study of bioactive compounds of Withania somnifera extract against topoisomerase iv type b. Proc. Natl. Acad. Sci. India Sect. B Biol. Sci. 2019, 90, 1-10, https://doi.org/10.1007/s40011-019-01110-z.

24. Umesh, H.R.; Ramesh, K.V.; Devaraju, K.S. Molecular docking studies of phytochemicals against trehalose6-phosphate phosphatases of pathogenic microbes. Beni-Seuf Univ. J. Appl. Sci. 2020, 9, 1-14, https://doi.org/10.1186/s43088-019-0028-6. 
25. Kumari, R.; Mishra, R.C.; Sheoran, R.; Yadav, J.P. Fractionation of antimicrobial compounds from Acacia nilotica twig extract against oral pathogens. Biointerface Res. Appl. Chem. 2020, 10, 7097-7105, https://doi.org/10.33263/BRIAC106.70977105.

26. Daina, A.; Michielin, O.; Zoete, V. SwissADME: a free web tool to evaluate pharmacokinetics, drug-likeness and medicinal chemistry friendliness of small molecules. Sci. Rep. 2017, 7, 42717, https://doi.org/10.1038/srep42717.

27. Mishra, R.C.; Kumari, R.; Yadav, S.; Yadav, J.P. Target based virtual screening of new leads inhibitor against bacterial cell division protein ftsz for the discovery of antibacterial agents. Med. Chem. 2020, 16, 169-175, https://doi.org/10.2174/1573406415666190206233448.

28. Hamedi, A.; Zengin, G.; Aktumsek, A.; Selamoglu, Z.; Pasdaran, A. In vitro and in silico approach to determine neuroprotective properties of iridoid glycosides from aerial parts of Scrophularia amplexicaulis by investigating their cholinesterase inhibition and antioxidant activities. Biointerface Res. Appl. Chem. 2020, 10, 5429-5454, https://doi.org/10.33263/BRIAC103.429454.

29. Arba, M.; Nurmawati, O. Identification of potential inhibitors for Bruton's Tyrosine Kinase (BTK) based on pharmacophore-based virtual screening. Biointerface Res. Appl. Chem. 2020, 10, 5472-5477, https://doi.org/10.33263/BRIAC103.472477.

30. Mishra, R.C.; Kumari, R.; Yadav, J.P. Comparative anti-dandruff efficacy of plant extracts prepared from conventional and supercritical fluid extraction method and chemical profiling using GCMS. J. Dermatolog. Treat. 2020, 31, 1-7, https://doiomparat.org/10.1080/09546634.2020.1799919.

31. Schlemmer, K.B.; Jesus, F.P.K.; Tondolo, J.S.M.; Weiblen, C.; Azevedo, M.I.; Machado, V.S.; Botton, S.A.; Alves, S.H.; Santurio, J.M. In vitro activity of carvacrol, cinnamaldehyde and thymol combined with antifungals against Malassezia pachydermatis. J. Mycol. Med.2019, 29, 375377,https://doi.org/10.1016/j.mycmed.2019.08.003.

32. Nejad, S.M.; Ozgunes, H.; Basaran, N. Pharmacological and toxicological properties of eugenol. Turk. J. Pharm. Sci. 2017, 14, 201-206, https://doi.org/10.4274/tjps.62207.

33. da Silva, I.C.; de Pontes, S.H.B.; Cavalcanti, Y.W.; Nonaka, C.F.; de Sousa, S.A.; de Castro, R.D. Antifungal activity of eugenol and its association with nystatin on Candida albicans. Pesqui. Bras. Odontopediatria Clin. Integr. 2017, 17, 1-8, https://doi.org/10.4034/PBOCI.2017.171.16.

34. Amiel, E.; Ofir, R.; Dudai, N.; Soloway, E.; Rabinsky, T.; Rachmilevitch, S. $\beta$-Caryophyllene, a compound isolated from the biblical balm of gilead (Commiphora gileadensis), is a selective apoptosis inducer for tumor cell lines. Evid. Based Complement. Alternat. Med. 2012, 8, 872394, https://doi.org/10.1155/2012/872394.

35. Selestino, N.M.C.; Vittorazzi, C.; Guimarães, A.C.; Martins, J.D.; Fronza, M.; Endringer, D.C.; Scherer, R. Effects of $\beta$-caryophyllene and Murraya paniculata essential oil in the murine hepatoma cells and in the bacteria and fungi 24-h time-kill curve studies. Pharm. Biol. 2017, 55, 190-197, https://doi.org/10.1080/13880209.2016.1254251.

36. Park, S.Y.; Choi, S.J.; Park, H.J.; Ma, S.Y.; Moon, Y.I.; Park, S.K.; Jung, M.Y. Hexane extract of green tea (Camellia sinensis) leaves is an exceptionally rich source of squalene. Food Sci. Biotechnol. 2020, 29, 76975, https://doi.org/10.1007/s10068-019-00724-3.

37. Aboody, M.S.; Mickymaray, S. Anti-fungal efficacy and mechanisms of flavonoids. Antibiotics 2020, 9, 45, https://doi.org/10.3390/antibiotics9020045.

38. Rocha, M.F.G.; Sales, J.A.; da Rocha, M.G.; Galdino, L.M.; de Aguiar, L.; Pereira-Neto, W.D.A.; de Aguiar Cordeiro, R.; Castelo-Branco, D.D.S.C.M.; Sidrim, J.J.C.; Brilhante, R.S.N. Antifungal effects of the flavonoids kaempferol and quercetin: A possible alternative for the control of fungal biofilms. Biofouling 2019, 35, 320-328, https://doi.org/10.1080/08927014.2019.1604948.

39. Kulkarni, M.; Hastak, V.; Jadhav, V.; Date, A.A. Fenugreek leaf extract and its gel formulation show activity against Malassezia furfur. ASSAY Drug Dev. Techn. 2020, 18, 45-55, https://doi.org/10.1089/adt.2019.918.

40. Teke, G.N.; Elisée, K.N.; Roger, K.J. Chemical composition, antimicrobial properties and toxicity evaluation of the essential oil of Cupressus lusitanica Mill. leaves from Cameroon. BMC Complem. Altern. M. 2013, 13, 1-9, https://doi.org/10.1186/1472-6882-13-130.

41. Xie, Y.; Wang, Z.; Huang, Q.; Zhang, D. Antifungal activity of several essential oils and major components against wood-rot fungi. Ind. Crops Prod. 2017, 108, 278-285, https://doi.org/10.1016/j.indcrop.2017.06.041.

42. Pereira, F.G.; Marquete, R.; Domingos, L.T.; Rocha, M.E.; Ferreira-Pereira, A.; Mansur, E.; Moreira, D.L. Antifungal activities of the essential oil and its fractions rich in sesquiterpenes from leaves of Casearia sylvestris Sw. Anais da Academia Brasileira. Ciencias 2017, 89, 2817-2824, https://doi.org/10.1590/00013765201720170339. 
43. Alves, F.A.; Morais, S.M.; Sobrinho, A.C.; Silva, I.N.; Martins, C. ;G.; Silva, A.A.; Fontenelle, R.O. Chemical composition, antioxidant and antifungal activities of essential oils and extracts from Plectranthus spp. against dermatophytes fungi. Rev. Bras. Saúde Prod. Anim. 2018, 19, 105115,http://dx.doi.org/10.1590/s1519-99402018000100010.

44. Li, L.; Seeram, N.P. Maple syrup phytochemicals include lignans, coumarins, a stilbene, and other previously unreported antioxidant phenolic compounds. J. Agr. Food Chem. 2010, 58, 11673-11679, https://doi.org/10.1021/jf1033398.

45. Lipinski, C.A.; Lombardo, F.; Dominy, B.W.; Feeney, P.J. Experimental and computational approaches to estimate solubility and permeability in drug discovery and development settings. Adv. Drug Deliv. Rev. 1997, 23, 3-25, https://doi.org/10.1016/S0169-409X(96)00423-1.

46. Fatonah, A.; Tambunan, U.S.F.; Pamungkas, W.O.; Dewanto, G.L.; Wicaksono, I.S. Discovery of GPX4 inhibitor by molecular docking simulation as a potential ferroptosis inducer. Biointerface Res. Appl. Chem. 2020, 10, 4929-4933,https://doi.org/10.33263/BRIAC101.929933.

47. Guo, S.; Huang, W.; Zhang, J.; Wang, Y. Novel inhibitor against Malassezia globosa LIP1 (SMG1), a potential anti-dandruff target. Bioorg. Med. Chem. Lett. 2015, 25, 3464-3467, https://doi.org/10.1016/j.bmcl.2015.07.005.

48. Sirisha, G.V.; Rachel, K.V.; Zaveri, K.; Yarla, N.S.; Kiranmayi, P.; Ganash, M.; Alkreathy, H.M.; Rajeh, N.; Ashraf, G.M. Molecular docking and in vitro studies of soap nut trypsin inhibitor (SNTI) against phospholipase A2 isoforms in therapeutic intervention of inflammatory diseases. Int. J. Biol. Macromol. 2018, 114, 556-564, https://doi.org/10.1016/j.ijbiomac.2018.03.139.

49. Ali, S.; Khan, F.I.; Mohammad, T.; Lan, D.; Hassan, M.; Wang, Y. Identification and evaluation of inhibitors of lipase from Malassezia restricta using virtual high-throughput screening and molecular dynamics studies. Int. J. Mol. Sci. 2019, 20, 1-19, https://doi.org/10.3390/ijms20040884.

50. Mishra, R.K.; Mishra, V.; Pandey, A.; Tiwari, A.K.; Pandey, H.; Sharma, S.; Pandey, A.C.; Dikshit, A. Exploration of anti-Malassezia potential of Nyctanthes arbor-tristis L. and their application to combat the infection caused by Mala s1 a novel allergen. BMC Complement Altern. Med. 2016, 16, 1-14, https://doi.org/10.1186/s12906-016-1092-2. 